\title{
Overview of Telemedicine Services in Mongolia
}

\author{
D. Enkhmaa ${ }^{1}$ (D) B. Munkhuu ${ }^{1} \cdot$ T. Baatar ${ }^{2} \cdot$ J. Purevsuren $^{1} \cdot$ E. Minjuur ${ }^{1} \cdot$ G. Luuzanbadam $^{1} \cdot$ R. MacKenzie ${ }^{3}$. \\ D. Warburton ${ }^{3} \cdot$ Sh. Enkhtur ${ }^{1}$
}

Accepted: 29 June 2021 / Published online: 9 July 2021

(C) The Author(s), under exclusive licence to Springer Science+Business Media, LLC, part of Springer Nature 2021

\begin{abstract}
Purpose of Review To highlight some achievements made through usage of telecommunication technologies in Mongolia as an example of a developing country with remote areas.

Recent Findings Telemedicine can be implemented not only for remote monitoring, diagnosing, and treating purposes but also can be effectively used for delivering knowledge and consultation services via modern technologies.

Summary Tele-health services can be successfully added to the traditional approach of physicians, especially in the countries with a vast territory with distant areas.
\end{abstract}

Keywords Telemedicine $\cdot$ Developing country $\cdot$ Telecommunication $\cdot$ Tele-health services $\cdot$ Mongolia

\section{Introduction}

Telemedicine is the use of information and communications technology to provide healthcare services to individuals for convenience or who are at some distance from the healthcare providers. It is determined as "the delivery of healthcare services, where distance is a critical factor, by all healthcare professionals using information and communication technologies for the exchange of valid information for diagnosis, treatment, and prevention of disease and injuries and research and evaluation and for the continuing education of healthcare providers, all in the interests of advancing the health of individuals and their communities" [1]. The low population density of 2 persons per square kilometer in Mongolia makes it challenging to deliver health services to rural and remote areas. Under Soviet rule, a regional and national system of air transport had been developed for urgent and emergent care.

This article is part of the Topical Collection on Technology and Pediatrics

D. Enkhmaa

dv.enkhmaa@gmail.com

1 National Center for Maternal and Child Health, Ulaanbaatar, Mongolia

2 UNFPA Country Office, Ulaanbaatar, Mongolia

3 Children's Hospital Los Angeles, Los Angeles, CA, USA
Although effective, it was not efficient, nor did it support the building of knowledge and skills of local healthcare workers. Furthermore, it thus became difficult to maintain qualified specialist facility care in these areas. This system collapsed with the dissolution of the Soviet Union in 1991. Thus, telemedicine interventions, including tele-consultations, information and communication technologies for communication in healthcare, internet-based interventions for diagnosis and treatment, tele-surgeries, tele-conferences, and tele-Cyber Academy education courses were very appealing to Mongolia to build the capacity of healthcare providers in rural areas and assist them in delivering quality health services.

Mongolia is a vast territory, roughly $1 / 6$ the size of the USA and with a population of 3,325,000 (only $1 \%$ of the USA), $67 \%$ urban and $33 \%$ rural. Thus, telemedicine is very practical for primary and secondary healthcare providers. In recent years, telemedicine with network technologies, video conferencing, and computer-based equipment have been introduced although in some instances, due to funding limitations, older approaches such as telephone and email are common. This paper will summarize our experience to demonstrate its effectiveness therapeutically while noting its technical limitations in our largely rural and low-tech settings. Reviews have concluded that there is relatively convincing evidence of effectiveness only for tele-radiology, tele-neurosurgery, tele-psychiatry, transmission of echocardiographic images, and the use of electronic referrals enabling email consultations and video conferencing between primary and 
secondary healthcare providers. Economic analyses suggested that tele-radiology, especially transmission of computed tomography (CT) images, can be cost-saving [2], while new questions beyond those of clinical and cost-effectiveness arise, pointing the need for larger studies of telemedicine as controlled interventions [3].

Due to its geographic challenges, the dissolution of the Soviet Union, and the loss of air transport options, Mongolia was relatively early in its explorations of alternatives. The pioneering work in introducing telemedicine in Mongolia was conducted by the California - Mongolia Medical program, a collaborative program between the National Center for Maternal and Child Health (NCMCH) and Children's Hospital Los Angeles (CHLA). In 2002, Mongolia was very rudimentary in its technological supportive resources as was knowledge about its application. Early assessment of telecommunication infrastructure, existing networks, support, and independent initiatives led to the development of a preliminary proposal of a telemedicine/consultation project to join up aimags (provinces) with the capital Ulaanbaatar and with the USA (CHLA).

The first network in clinical decision-making was introduced in field of heart disease management in 2003 with the support provided by the Government of the Grand Duchy of Luxembourg, and it was later expanded to the field of maternal and newborn health in 2007. This was a second project funded jointly by the Government of Luxembourg and UNFPA to support the Government Millennium Development Goals (MDGs) in reducing maternal and newborn morbidities and mortalities. With this initiative, the fully functional maternal and newborn tele-consultation network was established at $\mathrm{NCMCH}$ to build the capacity of the expert team at the reference center to provide clinical decisionmaking support for rural physicians in delivering quality case management to remote populations [3].

Additionally, in 2007, the Swiss Mongolian Pediatric Project (SMOPP), a humanitarian aid project, introduced Graf's method of hip ultrasound to Mongolia. The teleconsultations were based on web-based telemedicine network platforms mnObstetrics, which then expanded to all the provinces of Mongolia, reaching nationwide coverage as a result of these projects. Through the network, more than 1,824 teleconsultations were held, having been requested by provincial healthcare providers, which resulted in the reduction of unnecessary referrals by $8 \%$ on maternal and newborn health [4]. The most common complications requiring second opinions were pregnancy and childbirth complications associated with maternal medical conditions, where diseases of circulatory and respiratory systems dominated in maternal complications followed by fetal abnormalities [4]. Overall the rate of newborn screening coverage for developmental dysplasia of the hip in newborns was $76.7 \%$ [5•].
In addition to maternal and newborn health and ultrasound screening of the developmental dysplasia of the hip in newborns, the current most active telemedicine projects/services at $\mathrm{NCMCH}$ include pediatric surgery, tele-cervicography, and pediatric and neonatal intensive care. Tele-health services are implemented through [1] diagnosis and treatment of the patients at a distance, [2] tele-mentoring, [3] continuous medical education, [4] tele-conferences, $\left[5^{\bullet}\right]$ hands-on telemedicine training, [6•] case discussions (on maternal near miss and mortality, rare cases, avoidable near miss situations, etc.), and [7•] international tele-trainings. Consultations are using the web-based platforms by transferring patient's medical records, $x$-ray images, laboratory, and ECG test results. Tele-mentoring is performed by an expert that instructs, guides, and/or teaches another individual, usually less skilled in that field or specific procedure, from a remote location via a live audio and/or video feed [6॰].

In 2017, NCMCH received the prestigious United Nations Public Service Award (UNPSA) gathering 1st place in the category of "Innovation and Excellence in Delivering Health Services" for the work in implementing the telemedicine projects in Mongolia. The UNPSA is an annual competition, promoting the role, professionalism, and visibility of public service. Most notably, the telemedicine project contributed to the overall reduction of maternal mortality in the country by 2/3, making Mongolia one of only 9 countries in the world to reach its targets for the MDGs by the end of 2015.

The NCMCH is one of eight first place winners, selected from 552 nominations, and one of the 12 institutions from 11 countries to be recognized at the UNPSA 2017 for public service initiatives aligned with the 2030 Agenda global Sustainable Development Goals.

This review paper describes some achievements of telemedicine service in Mongolia, specifically implemented at $\mathrm{NCMCH}$ as a main partner and coordinating institution of the Government of Mongolia.

\section{Improved Capacity in Digital Technology Use}

With implementation of Maternal Child Health (MCH) telemedicine project, modern diagnostic and telemedicine equipment such as two- and three-dimensional digital ultrasound machines with DICOM image processing and high-speed computer with audio visual accessories connected with internet and intra-partum cardiotocography monitoring with central station and digital colposcopes were provided. The software platform was Campus Medicos and MnObstetrics which are collaborative platforms for exchange of medical knowledge, distance consultations, clinical forums, and distant teaching in medicine. Images can be uploaded in series, and shared videos allow doctors to view the same live scenario, talk to each other, write chats, and store images in a patient case. Expert doctors at the reference center write their diagnosis as the first or second opinion [6॰]. 
Telemedicine projects, implemented at NCMCH with other remote areas, aimed at upgrading the diagnostic and medical equipment, organizing serial clinical trainings for service providers on new and innovative approaches and techniques through updated clinical reference materials, sensitizing the national and provincial governments and health administration, and enhancing professional networking between participating hospitals, national institutions, and international partners [3].

Tele-conferences involving physicians nationwide, being held in Ulaanbaatar, enable professionals to discuss practical problems and scientific evidence at national and international levels.

In 2013, an international tele-link was established between the NCMCH and CHLA partially supported by a gift from the Jennifer Lopez Foundation. This allowed the advanced subspecialty pediatric and adolescent consultation of a premier American children's hospital to be accessed live by the professional staff of NCMCH. Problem cases in diagnosis and management were presented in case conference style which led to not only advances in patient care but also in education of the attending medical professionals in attendance.

In addition, the Mongolian National University of Medical Sciences has established a Cyber Academy for distance postgraduate education. Over 500 advanced students have signed up from among a suite of 15 online master's degrees in such subjects as general medicine, environmental sciences, child health, and so on. Courses are given bilingually in Mongolian and English. Students may access the courses and proceed at their own pace of online remote learning. Students have signed up for the online Cyber academy from across the Mongolian speaking regions of Mongolia, China, and southern Siberia, Russia.

\section{Access to Quality Maternal and Newborn Healthcare Improved}

a. Nationwide tele-consultation network is available and accessible for quality $\mathrm{MCH}$ case management: As reported in the $\mathrm{MCH}$ project evaluation [3], over 1800 mothers and newborns' lives are saved through the network access. A distance communication of specialists between the reference and local hospitals via consultation cases has obviously improved and resulted in increased strategic clinical judgment of local service providers for patient management.

b. Improved human resource capacity in maternal and newborn care among participating hospitals: With introduction of new training methodology using clinical simulations as an international best practice in upgrading of service providers' hands-on skills in high-risk obstetrics and emergency obstetrics, the quality of care in local hospitals was improved significantly. For instance, [1] as a result capacity development, the quality of diagnostics of rural hospitals was improved as $88.3 \%$ of all consulted cases were managed locally according to advise provided by the experts [6•], and [2] in the last content analysis done in 2015 , the proportion of agreement in the diagnosis between the provincial specialist and $\mathrm{NCMCH}$ expert reached $85.4 \%$ as compared to baseline of $62.3 \%$ [7•]. Moreover, the percentage of the locally managed cases out of the total consulted cases accounted for $86 \%$, and the percentage of referred cases to upper level care in Ulaanbaatar city accounted for $14.1 \%$ [8].

\section{Access to Continuous Medical Education}

To support the access to continuous medical education by rural physicians, the project developed an e-learning platform (http://elearning.obgyn-telemedicine.mn/).To ensure the government ownership and sustainability of capacity development interventions, 4-week training modules are integrated into the Continuous Medical Education (CME) program of the Center for Health Development and are offered on a regular basis to specialists as two CME credit hour courses. To date, a total of 249 specialists successfully registered as active users and received on-line credits while completing all modules. To support professional networking between specialists from various national and international institutions, the national event "Improving Practice by Exchanging" was introduced as a National Congress [3, 6•].

\section{Newborn Ultrasound Screening for Developmental Dysplasia of the Hip}

Developmental dysplasia of the hip is a major health problem which can lead to lifelong treated or handicapped individuals if diagnosis is missed in first weeks of life. Mongolia is the first Asian country that launched the universal ultrasound hip screening with the help of SMOPP project. The Mongolian government approved a nationwide screening program to offer ultrasound hip screening by Graf technique for all newborns, after results of prospective birth cohort study [9॰] revealed that hip ultrasound is feasible at $\mathrm{NCMCH}$ and that the incidence of developmental hip dysplasia (DDH) in neonates is comparable with that in European neonates (1 to 2\%). Moreover, it was shown that early ultrasound-based assessment and a simple flexion-abduction treatment of DDH with monthly follow-up ultrasound controls until healing leads to maturation of the hip joints in all cases [9•]. Mongolian Ministry of Health decided to initiate the ultrasound screening for DDH in newborns throughout Ulaanbaatar, the capital 
city; and subsequently it was expanded step by step as a nationwide screening program since 2010 with the help of the SMOPP project. Pediatricians and neonatologists were taught the theory and practical application of the examination by ultrasound, where the screeners received face-to-face basic and advanced training for ultrasound of the hip and continuous support from experts at $\mathrm{NCMCH}$, and further quality was checked by Mongolian and Swiss supervisors.

Since multicenter hospitals are involved in the screening, a web-based, password-protected quality tool "HIPSCREEN" enables screeners to upload 4 images (two per hip side) as DICOM files exported from the ultrasound device by the screeners. This allows continuous and reliable review of hip grading, diagnosis of DDH, and treatment decisions. Also, hip ultrasound screening coordinators at the $\mathrm{NCMCH}$ perform weekly and monthly checks of the screening density and quality of each maternity hospital in Ulaanbaatar and rural aimags. Regular visits or phone calls are made to individual sites. Furthermore, screeners receive regularly refresher training in screening methods. These factors all have a positive impact on the screening coverage and quality of diagnoses and treatment. This 4 eyes quality control system enables a matchless and unique optimization of diagnosis and therapy. The hip ultrasound screening coverage rate in newborns nationwide increased from $73.6 \%$ in 2017 to $82.1 \%$ in 2019 [5•], showing that tele-services have been successfully implemented in this area.

\section{Pediatric Surgery}

Current applications for tele-mentoring in pediatric surgery tend to include rare, technically challenging cases that are approached in a minimally invasive fashion (i.e., laparoscopy, thoracoscopy) where the primary surgeon has suboptimal experience with the technique.

Recently, this kind of telemedicine is developing for pediatric surgeons to surgeons in the country. Indications of telementoring are the following: (a) emergency surgery care for pediatric surgical diseases in secondary healthcare hospitals; (b) a hospital, in which there are pediatric surgeons, pediatric anesthesiologists and intensivists, neonatologists, and pediatric nurses, and however they have suboptimal experience with individual technique; and (c) area with COVID-19 infection and other pandemics; it is not possible to transfer the patient to a referral hospital, and there is risk to transfer.

In 2020, our pediatric surgeons did tele-mentoring for 8 cases with intestinal intussusception, congenital duodenal atresia, anal atresia and congenital inguinal hernia, and congenital abdominal wall defects who were located in very long distance places (Gobi-Altai, Bayan-Ulgii, Khovd, Khuvsgul, Bayankhongor, and Dundgovi provinces) from Ulaanbaatar. The expert was able to guide the novice through a procedure in which they previously had minimal experience. In 2021, the surgeons have done tele-mentoring for 3 cases. For the latest 5 years, we did video consultation to primary and secondary healthcare providers in all 21 prefectures, and they received consultation for 14 kinds of specialists (pediatric intensive care, tele-radiology such as CT, echographic image of hip in neonates, surgery, respiratory disease, neonatalogy, neurology, onco-hematology, nephrology, endocrinology, cardiology, gastroenterology, and so on). Of them, specialist for intensive care, obstetrician and gynecologist, and radiologist were the most common, and they give medical consultation by telemedicine the most frequently.

\section{Tele-cervicography}

Early diagnosis of cervical cancer is critical to reduce complications and mortality. Even though colposcopy is the most appropriate procedure for evaluating the patients with abnormal cytology, specific instrument and experienced colposcopist [10] make this procedure expensive. Cervicography was introduced by Stafl in 1981 [11•] to ameliorate the problems of colposcopy and has been widely used as a screening tool for cervical cancer since. It has been reported that cervicography possibly be used as a mass screening method by its comparable simplicity and accuracy [12]. Procedures such as taking 35-mm photographs of the cervix and sending the results to the doctors used to be expensive and time-consuming. Recently, digital cervicography adopted digital pictures and thus saved the cost and time for interpretation of abnormal cervix. With the aid of digital system, telecervicography is being developed where utilization of digital cameras linked to the internet enables health workers in remote areas to capture cervical images and transmit them to experts for further opinion. Project on tele-cervicography has been introduced to Mongolia in 2017 as a joint project of the Government of Mongolia and Korea. NCMCH is serving as a main coordinator of this project. There are three main regional diagnostic and treatment centers that have been collaborating, and a total 8681 women underwent tele-cervicoscopy in 2017-2020, of which $44.7 \%$ were from rural areas. Cancer was detected in $1.02 \%$ who referred to the National Cancer Center for further treatment [13].

\section{Discussion}

Telemedicine has been successfully implemented in Mongolia in different areas of health over the last 2 decades thanks to high commitment of different donors and implementing partners including national team. Networking between rural and urban health professionals has widely been improved based on telecommunication channels. A distance communication 
of specialists between the reference and local hospitals via tele-consultation cases clearly improved and resulted in increased strategic clinical judgment of local service providers for patient management. Tele-conferences are being regularly held in all areas involving local and international experts. Not only networking, but also high-quality equipment was procured to rural hospitals, especially in the area of maternal and newborn health enabling physicians to perform full series screening of obstetric ultrasound diagnosis including fetal abnormality and placental and vascular conditions in early age of gestation. Improved intra-partum fetal monitoring and a number of major surgical cases were increased in rural hospitals [4]. Emergency obstetrics care in the participating hospitals was upgraded to complement quality and accessibility for the rural population with introduction of international best practice guidelines accepted by the Asia and Oceania Federation of Obstetrics and Gynecology.

Mongolia is the first Asian country to launch a program of universal ultrasound hip screening for DDH in newborns. Based on the results of several studies, the Mongolian government approved a nationwide screening program that required all maternity hospitals to offer ultrasound hip screening by Graf technique for all newborns. Trained neonatologists or pediatricians perform the hip ultrasound screening following standardized procedures. Screening, follow-up monitoring, and flexion-abduction treatment are offered free of charge under the supervision of experts. The goal of the program is to screen every newborn baby using hip ultrasound and to provide early preventive treatment to eliminate disability due to DDH. As, we aim to determine its coverage and treatment rate. Recent studies demonstrated that a population-based ultrasound screening program for DDH in children is feasible with coverage of $81.2 \%$ even though the screening was comparatively new to both the general population and to healthcare professionals $[5 \cdot]$.

Rare and complicated surgical cases are being managed in rural hospitals under the strict monitoring and guidance from experts via telecommunication. Video consultations to primary and secondary healthcare providers in all 21 prefectures are being held constantly with the most upgraded guidance and information.

Introduction of tele-cervicography avoids women from unnecessary travel distance from remote area and serves as an adjunct diagnostic tool for cervical abnormality.

It should be noted that there is still room for improvement in telemedicine in Mongolia in accordance to major aspects and recommendations based on case studies [14]. We highlighted several areas where telemedicine is directly experienced and successfully implemented and overall health services provided are generally improved.

Challenges related to implementation of telemedicine in developing countries are described elsewhere [14••, 15••]. High cost of telemedicine system, resistance to change and slow clinical acceptance of telemedicine, unavailability of the required ICT infrastructure for telemedicine (e.g., internet connection and bandwidth for high speed telecommunications) especially in rural areas, and lack of standards, mainly for data exchange security, safety, and privacy, were the major challenges. Broadening the telemedicine to integrate the technologies as standard part of health services might help to overcome above challenges. The Government of Mongolia and the International Development Agency of the World Bank had signed Financing Agreement in 2015 for implementation of e-health project during 2015-2020 which was officially launched in 2016. Currently it has three components that include [1] building the e-health integration system, [2] institutional strengthening and capacity building, and [3] project management. Quality of healthcare in developing countries can be improved by introducing modern technologies into health system through telemedicine and e-health.

\section{Conclusion}

Telemedicine and education services are being successfully and effectively implemented in several areas of health in Mongolia. Exposure to tele-health service models can further improve rural service capabilities in the future.

Abbreviations UNFPA, United Nations Population Fund; UNICEF, United Nations Children 's Fund; DICOM, Digital Imaging and Communications in Medicine; NCMCH, National Center for Maternal and Child Health; CHLA, Children's Hospital Los Angeles; MDGs, Millennium Development Goals; SMOPP, Swiss Mongolian Pediatric Project; UNPSA, United Nations Public Service Award; $\mathrm{MCH}$, Maternal and Child Health; CME, Continuous Medical Education; DDH, Developmental Hip Dysplasia; WHO, World Health Organization

Acknowledgements We would like to express our appreciation to donors and international organizations such as Government of Luxemburg, Government of Korea, UNFPA, WHO, and UNICEF for their support in introduction of telemedicine services in Mongolia.

\section{References}

Papers of particular interest, published recently, have been highlighted as:

- Of importance

•• Of major importance

1. Organization WH. Telemedicine: opportunities and developments in Member States: report on the second global survey on eHealth. Global Observatory for eHealth Series. 2009;2.

2. Roine R, Ohinmaa A, Hailey D. Assessing telemedicine: a systematic review of the literature. CMAJ: Canadian Medical Association journal = journal de 1 ' Association medicale canadienne. 2001;165(6):765-71. 
3. Ekeland AG, Bowes A, Flottorp S. Effectiveness of telemedicine: a systematic review of reviews. Int J Med Inform. 2010;79(11):73671.

4. B T. Access to quality $\mathrm{MCH} / \mathrm{RH}$ and newborn health care is improved and expanded. UNFPA; 2017.

5. Ulziibat M, Munkhuu B, Schmid R, Baumann T, Essig S. Implementation of a nationwide universal ultrasound screening programme for developmental dysplasia of the neonatal hip in Mongolia. J Child Orthop. 2020;14(4):273-80 Describes in detail the results of national coverage of nenonatal dysplasia of the hip.

6. Harting MT, Wheeler A, Ponsky T, Nwomeh B, Snyder CL, Bruns NE, et al. Telemedicine in pediatric surgery. J Pediatr Surg. 2019;54(3):587-94 Describes the current state of telemedicine in surgery and pediatric surgery, and provide basic themes for successful implementation of a pediatric surgical telemedicine program.

7. Baatar T SN, Bayanbileg S, Dorjsuren Kh. Telemedicine support for Maternal and newborn health support: analysis of content in communication. Global Telemedicine and eHealth Updates, Medetel 2013. Describes the progress made in maternal and newborn health in remote area after implementing telelmedicine project.

8. Baatar T, Suldsuren N, Bayanbileg S, Seded K. Telemedicine support of maternal and newborn health to remote provinces of Mongolia. Studies in health technology and informatics. 2012;182:27-35.
9. Munkhuu B, Essig S, Renchinnyam E, Schmid R, Wilhelm C, Bohlius J, et al. Incidence and treatment of developmental hip dysplasia in Mongolia: a prospective cohort study. PLoS One. 2013;8(10):e79427 First to report in the peer reviewed journal the incidence of newborn hip dysplasia.

10. Chase DM, Kalouyan M, DiSaia PJ. Colposcopy to evaluate abnormal cervical cytology in 2008. Am J Obstet Gynecol. 2009;200(5): 472-80.

11. Stafl A. Cervicography: a new method for cervical cancer detection. Am J Obstet Gynecol. 1981;139(7):815-25 First to introduce this method.

12. Ferris DG, Payne P, Frisch LE, Milner FH, di Paola FM, Petry LJ. Cervicography: adjunctive cervical cancer screening by primary care clinicians. The Journal of family practice. 1993;37(2):158-64.

13. NCMCH. Progress report on Telecervicography project. 2020.

14.• Combi C, Pozzani G, Pozzi G. Telemedicine for developing countries. A survey and some design issues. Appl Clin Inform. 2016;7(4):1025-50 Provides a recommendation and main aspects of telemedicine in developing country.

15.• Hsing JC, Wang CJ, Wise PH. Child health and telehealth in global, underresourced settings. Pediatr Clin N Am. 2020;67(4):773-81 Describes challenges of telemedicine in underresourced settings.

Publisher's Note Springer Nature remains neutral with regard to jurisdictional claims in published maps and institutional affiliations. 